\title{
Complete Visual Rehabilitation in a Patient with No Light Perception after Surgical Management of a Penetrating Open-Globe Injury: A Case Report
}

\author{
Tania A. Baban Fady K. Sammouh Haytham M. ElBallouz \\ Elias L. Warrak \\ Department of Ophthalmology, Saint George Hospital, University Medical Center, Beirut \\ Lebanon
}

\section{Key Words}

Ocular trauma - Open-globe injury - No light perception · Foreign body · Penetrating injury . Visual outcome $\cdot$ Middle East $\cdot$ Lebanon

\begin{abstract}
Open-globe injuries with no light perception (NLP) carry a poor prognosis, and many ophthalmologists select a primary enucleation or evisceration as a result. We present a case of complete visual rehabilitation in a patient who presented with NLP after a zone III penetrating open-globe injury. We recommend patient counseling regarding decision making and management of ocular trauma with NLP, since timely intervention and close follow-ups may restore useful or complete vision even in severely traumatized eyes.

(c) 2015 S. Karger AG, Basel
\end{abstract}

\section{Introduction}

Ocular trauma can result in significant visual morbidity [1,2]. To date, ocular trauma remains a subject of controversies and arguable strategies for the optimal management especially open-globe injuries (OGIs) [3]. No light perception (NLP) vision secondary to severe ocular injury characteristically carries a poor prognosis [4]. Surgical repair for severely traumatized eyes with NLP is a continuous debate [3] and these cases commonly end up with enucleation [4]. Furthermore, the rare risk of sympathetic ophthalmia often leaves spe- 
Baban et al: Complete Visual Rehabilitation in a Patient with No Light Perception after Surgical Management of a Penetrating Open-Globe Injury: A Case Report

cialists confronted with the critical decision of whether or not to repair severely traumatized eyes with limited or no visual prognosis [3].

Studies regarding chronic management, outcome and prognosis of NLP following OGI in the literature are limited $[1,2,5]$, particularly from the Middle East. We present a case of NLP vision as a result of a penetrating OGI, who had complete visual recovery following multiple surgical interventions.

\section{Case Report}

A previously healthy 16-year-old male patient, with no history of ocular disease, presented in February 2013 after sustaining a penetrating glass injury to his left eye (OS). He described an abrupt loss of vision in the affected eye and was first transferred to a hospital where the glass foreign body was removed manually in the emergency department by a nonspecialist, which possibly contributed to a further extension of his initial injury. Subsequently, he was found to have NLP vision in his OS on examination. A slit-lamp evaluation revealed a zone III injury (posterior OGI) with broad corneoscleral laceration extending posteriorly. A ruptured lens and protruding vitreous were also noted (fig. 1a). The patient had a distorted pupil in his OS, which was non-reactive to light (using the indirect ophthalmoscope light at the highest intensity). However, a consensual pupillary reaction was present in his right eye (OD; when light was shone on the injured globe). An initial B-scan at presentation showed a collapsed globe (fig. 3a). The patient was transferred to the operating room where he underwent surgical repair of the corneoscleral laceration under general anesthesia. The globe was first sutured at the limbus using nylon 10-0 sutures and a further dissection showed an extension of the laceration posteriorly toward the optic nerve. Cornea, sclera and then conjunctiva were all sutured carefully using nylon 10-0 sutures, and an eye patch was carefully placed. Oral ciprofloxacin $250 \mathrm{mg}$ twice per day and prednisone $50 \mathrm{mg}$ once daily were started.

On the first day after surgery, the eye patch was removed and an examination showed a retained globe integrity with cataract formation. The patient was started on topical Zymaxid (gatifloxacin) and Pred Forte (prednisolone acetate) every $2 \mathrm{~h}$.

The patient was noted to have an unchanged globe integrity with rapidly increasing cataract formation when followed up on postoperative days 4, 7, 14 and 30 (fig. 1b-d). The topical antibiotic and steroids were kept at 5 times per day from day 7 until day 14 postoperatively. Then, both were tapered and eventually stopped over the following month. At 1 week postoperatively, oral antibiotics were stopped and oral steroids were tapered gradually over 1 month. A follow-up B-scan revealed a flat retina and the patient was scheduled for regular monthly follow-ups. On day 7 postoperatively, the patient was found to have an uncorrected visual acuity (UCVA) in his OS of counting fingers near face with a reactive pupil to light and a normal consensual reflex in his OD. His UCVA worsened over the following follow-up visits as the cataract formation increased.

Two months after the initial surgery, a repeated B-scan showed a flat retina and a clear well-formed vitreous cavity (fig. 3b); accordingly, the patient was scheduled for cataract extraction. The anterior capsule was partially ruptured and fibrotic secondary to the penetrating injury. Yet, circular capsulorrhexis was managed to be done with the help of VisionBlue ${ }^{\circledR}(0.06 \%$ trypan blue ophthalmic solution). Phacoemulsification of the cataract was performed successfully, followed by an anterior vitrectomy to remove the protruding vitreous. A posterior chamber intraocular lens was implanted in the sulcus. The procedure was well tolerated and the affected eye was unpatched on the second day. The patient was start- 
ed on topical antibiotics and steroids and followed up on days 1, 3, 6, 21 (fig. 2a, b) and 6 weeks postoperatively during which the topical treatment was tapered gradually.

The patient reported a gradual improvement in his vision after cataract extraction. On the 3rd week after the second surgery, UCVA in his OS was 20/200 and on the 11th week, UCVA improved to $20 / 50$ with a best corrected visual acuity reaching $20 / 20$ with a manifest refraction: plano $+1.75 \times 45^{\circ}$ (fig. 2 c).

\section{Discussion}

Despite the advances in surgical techniques in ophthalmology over the past 2 decades, unresolved controversies regarding the optimal management of OGI remain. However, a posttraumatic visual acuity of NLP alone should no longer be an indication for primary enucleation or evisceration [1]. Numerous variables have been identified to significantly correlate with the final visual outcome in patients with OGI $[2,6,7]$. Surgical outcomes of injured eyes with poor vision have been reported with diverse success rates [4, 5, 8-10]. Andreoli and Andreoli [2] reported an overall worse postoperative visual acuity in patients requiring multiple procedures than in those who underwent vitreoretinal surgeries. To our knowledge, there have been no reported cases with a complete visual recovery as seen in our case, which highlights the fact that even in severely traumatized eyes with NLP, surgical intervention may restore vision. In our patient, there was no apparent direct traumatic optic nerve injury, and NLP vision may be related mainly to the disfigured, collapsed and hypotonic globe. The presence of a consensual pupillary reflex in the OD upon presentation supports the absence of traumatic optic neuropathy and may suggest a favorable prognosis later on. Also, the decrease in UCVA after the first week following the first surgery is essentially due to the significant cataract formation.

In conclusion, we suggest patient-specific counseling and decision making regarding the management of such scenarios since timely intervention and close follow-ups may restore useful or, as in our case complete vision, even in severely traumatized eyes where an enucleation seems inevitable. However, large multicenter, controlled, prospective studies would be ideal to evaluate the final visual outcomes in patients with NLP following surgical interventions in OGIs.

\section{Statement of Ethics}

A detailed consent form was obtained from the patient and the patient's next of kin.

\section{Disclosure Statement}

The authors have no financial interests to disclose.

\section{References}

1 Agrawal R, Wei HS, Teoh S: Predictive factors for final outcome of severely traumatized eyes with no light perception. BMC Ophthalmol 2012;12:16.

2 Andreoli MT, Andreoli CM: Surgical rehabilitation of the open globe injury patient. Am J Ophthalmol 2012;153:856-860. 
Baban et al:: Complete Visual Rehabilitation in a Patient with No Light Perception after Surgical Management of a Penetrating Open-Globe Injury: A Case Report

3 Agrawal R, Shah M, Mireskandari K, Yong GK: Controversies in ocular trauma classification and management: review. Int Ophthalmol 2013;33:435-445.

4 Morris R, Kuhn F, Witherspoon CD: Management of the opaque media eye with no light perception; in Alfaro DV III, Lieggett PE (eds): Vitreoretinal Surgery of the Injured Eye. Philadelphia, Lippincott-Raven, 1999, pp 113-124.

5 Salehi-Had H, Andreoli CM, Andreoli MT, Kloek CE, Mukai S: Visual outcomes of vitreoretinal surgery in eyes with severe open-globe injury presenting with no-light-perception vision. Graefes Arch Clin Exp Ophthalmol 2009;247:477-483.

6 Rahman I, Maino A, Devadason D, Leatherbarrow B: Open globe injuries: factors predictive of poor outcome. Eye 2006;20:1336-1341.

7 Rofail M, Lee GA, O’Rourke P: Prognostic indicators for open globe injury. Clin Experiment Ophthalmol 2006;34:783-786.

8 Heidari E, Taheri N: Surgical treatment of severely traumatized eyes with no light perception. Retina 2010;30:294-299.

9 Schmidt GW, Broman AT, Hindman HB, Grant MP: Vision survival after open globe injury predicted by classification and regression tree analysis. Ophthalmology 2008;115:202-209.

10 Agrawal R, Rao G, Naigaonkar R, Ou X, Desai S: Prognostic factors for vision outcome after surgical repair of open globe injuries. Indian J Ophthalmol 2011;59:465-470.
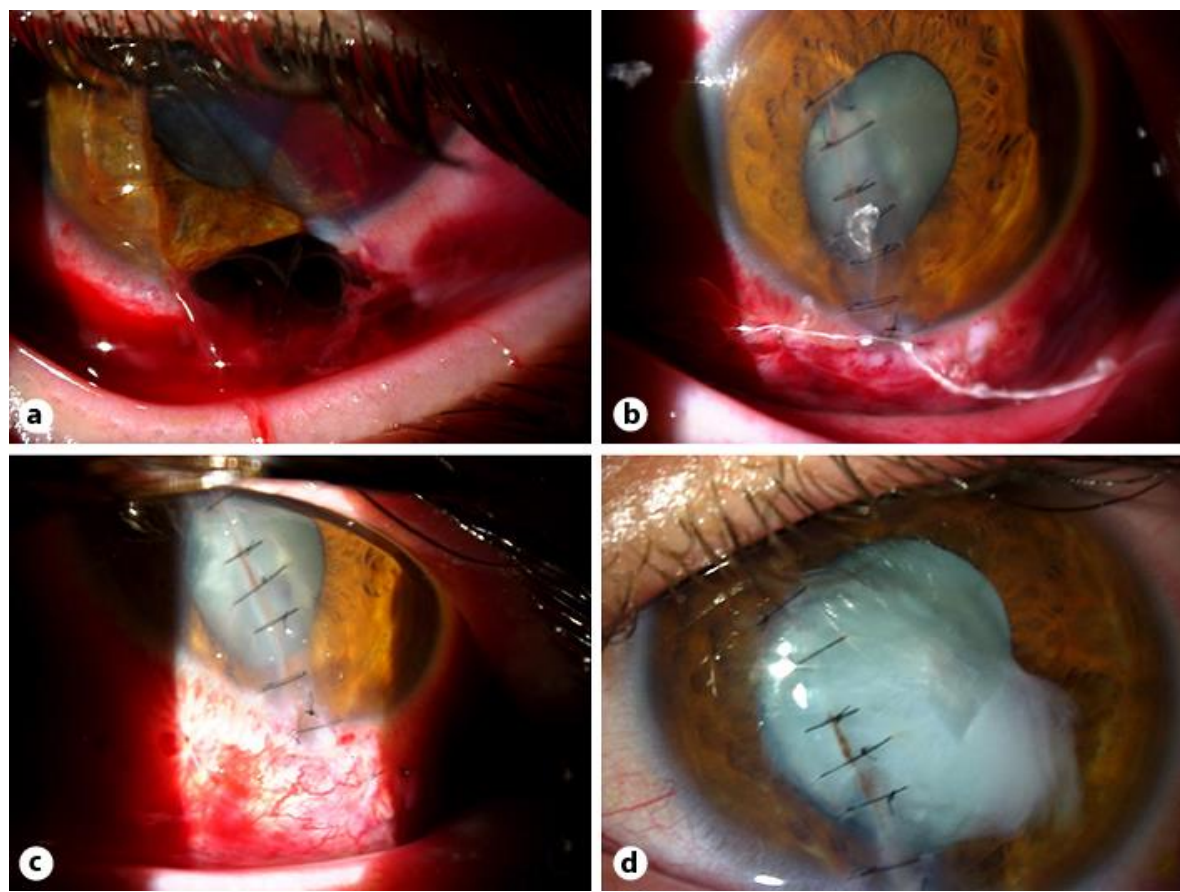

Fig. 1. a Ruptured globe and corneoscleral laceration extending posteriorly with prolapsed vitrious and iris at the initial presentation. b Day 4 postoperatively: the successful repair of the corneoscleral laceration. c Day 7 postoperatively: conjunctival healing over the scleral laceration with an increase in cataract formation noted. d One month postoperatively: good globe integrity with an irregular pupil and significant cataract formation. 
Baban et al.: Complete Visual Rehabilitation in a Patient with No Light Perception after Surgical Management of a Penetrating Open-Globe Injury: A Case Report
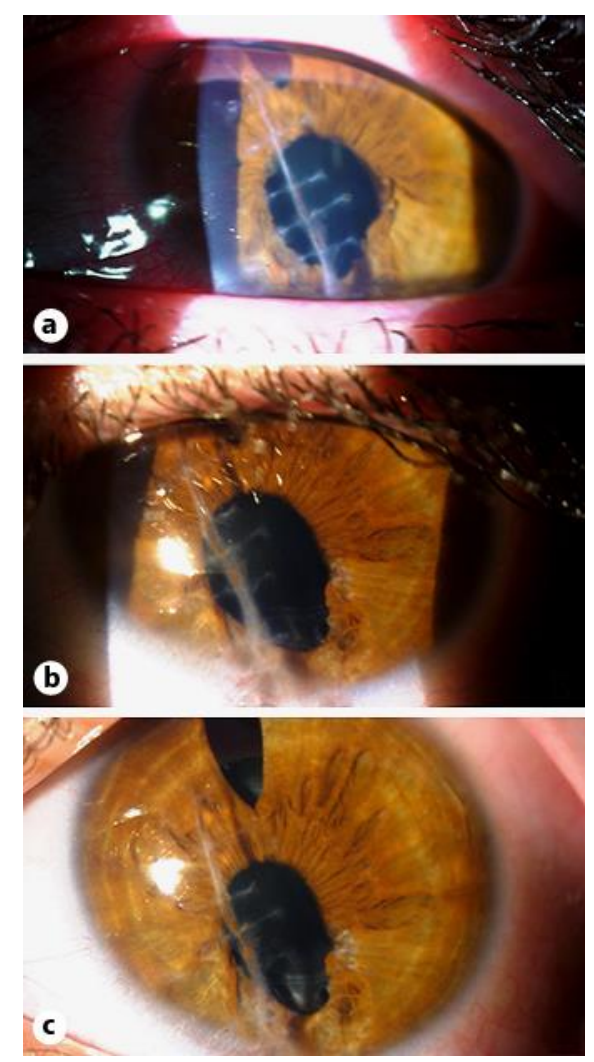

Fig. 2. a Day 1 after cataract extraction, anterior vitrectomy and peripheral iridectomy. Corneal sutures from the initial surgery were removed. $\mathbf{b}$ Three weeks after the second surgery: a good globe integrity with a notable corneal scar. c Six months after the initial presentation. 
Case Reports in

Ophthalmology

\begin{tabular}{l|l}
\hline \multicolumn{2}{l}{ Case Rep Ophthalmol 2015;6:204-209 } \\
\hline DOI: 10.1159/000434636 & $\begin{array}{l}\text { ○ 2015 S. Karger AG, Basel } \\
\text { www.karger.com/cop }\end{array}$ \\
\hline
\end{tabular}

Baban et al.: Complete Visual Rehabilitation in a Patient with No Light Perception after Surgical Management of a Penetrating Open-Globe Injury: A Case Report

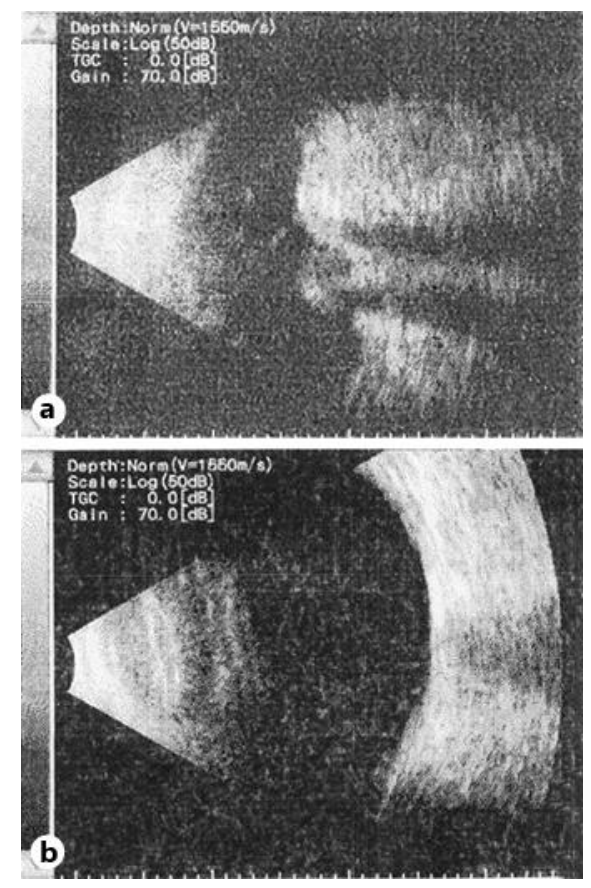

Fig. 3. a A B-scan upon initial presentation showing a complete globe collapse (flat tire sign). b A B-scan 2 months after the initial presentation showing a clear vitreous with an intact retina. 